\title{
Analysis of acoustic leak signals for enhancing sensitivity of control due to the creation of effective diagnostic indicators
}

\author{
Dmitry M. Shvetsov ${ }^{1}$, Evgeny L. Trykov ${ }^{1}$, Sergey T. Leskin ${ }^{2}$, Aleksey Yu. Puzakov² \\ 1 JSC "NTTS Diaprom", 14 Gasgoldermaya str., Moscow, 109518 Russia \\ 2 Obninsk Institute for Nuclear Power Engineering, National Research Nuclear University "MEPHI”, 1 Studgorodok, Obninsk, Kaluga Reg., \\ 249030 Russia \\ Corresponding author: Sergey T. Leskin(STLeskin@mephi.ru)
}

Academic editor: Elena Artyushkova Received 23 October 2018 Accepted 12 November 2018 Published 26 November 2018

Citation: Shvetsov DM, Trykov EL, Leskin ST, Puzakov AY (2018) Analysis of acoustic leak signals for enhancing sensitivity of control due to the creation of effective diagnostic indicators. Nuclear Energy and Technology 4(2): 141-147. https://doi.org/10.3897/ nucet.4.30775

\begin{abstract}
Acoustic leak control systems (for instance, SAKT) are used at present for controlling leak tightness of equipment and pipelines, as well as for detecting in timely manner coolant leaks from the primary cooling circuit of nuclear reactor installations (NRI) during operation of power unit on different power levels in the modes of normal operation and during disturbances of normal operation. Time averaged dispersion of acoustic signal is used as the main diagnostic indicator for detecting leaks in these systems. Sensitivity of this indicator is determined by the exceedance by the signal of the preset threshold value which is defined in accordance with the background. Here, background values of acoustic signal depend on the operational modes of the equipment and do not allow in many cases determining coolant leak during early stages of leak development.
\end{abstract}

New approach to the formation of diagnostic indicators for detecting loss of sealing in the circuit during early stage of development of coolant leak is suggested.

Methodology for obtaining diagnostic indicators is based on the processing in different frequency bands of acoustic signal accompanying coolant leakage from the pipeline using the method of principal components.

Efficiency of the developed methodology of coolant leak detection is illustrated by processing acoustic signals for experimental facility modeling coolant leakage in case of loss of sealing of the circuit.

Even in the presence of significant acoustic background sensitivity of the method allows detecting leaks with significantly lower flow rates (up to five times smaller) than the conventional processing of acoustic signals.

Implementation of the developed methodology will not require significant expenditures for upgrading already existing leak control systems operated at present on different NPPs.

\section{Keywords}

control of leak tightness of equipment; dispersion of acoustic signal; modeling coolant leaks; method of principal components; additional diagnostic indicators; pattern identification

* Russian text published: Izvestiya vuzov. Yadernaya Energetika (ISSN 0204-3327), 2018, n.1, pp. 112-121.

Copyright Shvetsov DM et al. This is an open access article distributed under the terms of the Creative Commons Attribution License (CC-BY 4.0), which permits unrestricted use, distribution, and reproduction in any medium, provided the original author and source are credited. 


\section{Introduction}

Acoustic leak control systems (SAKT in Russian abbreviation) are used at present for controlling leak tightness of equipment and pipelines, as well as for detecting in timely manner coolant leaks from the primary cooling circuit of nuclear reactor installations (NRI) during operation of power unit on different power levels in the modes of normal operation and during disturbances of normal operation (Arkadov et al. 2010, Weiss et al. 1988, Basseville 1988, Herbert 1984). Time averaged dispersion of acoustic signal is used as the main diagnostic indicator for detecting leaks in this system. Sensitivity of the above indicator is determined by the exceedance by the signal of the preset threshold value which, in turn, is determined by the background. Here, background values of acoustic signal depend on the operational modes of the equipment and do not allow in many cases determining coolant leak during early stages of leak development.

Additional diagnostic indicators and methods for their identification allowing more reliably determining presence of leakages in the case of small coolant leakage flow rates are examined in the present paper. Pattern identification theory (Abagyan et al. 1987, Tu and Gonsales 1978, Fukunaga 1972, Classification and clustering (1980), Tao Gu and Tou 1982) successfully applied in different studies on the diagnostics of NRI equipment (Leskin et al. 2016, Leskin et al. 2017, Ujita Hiroshi 1986, Fault diagnosis in dynamic systems 1989, Willsky 1976, Iserman 1984, Frank 1990, Reisen and Marshall 1988, Abagyan et al. 1987, Herbert 1984, Pavelko 1990, Urig Robert 1991, Leskin 1997) constitutes the basis for formation of additional diagnostic indicators.

\section{Experimental facility}

Measurements were conducted on the experimental stand imitating circulation loop at the INPE NRNU MEPhI designed for implementation of studies of acoustic noises and hydraulic characteristics of circulating coolants.

The experimental facility consists of the device for imitation of leak with compressed air and the simulator of heat exchange pipe intended for investigation of the nature of propagation of surface waves inside steel pipe. The simulator represents a pipe made of stainless steel $1 \mathrm{X} 18 \mathrm{H} 10 \mathrm{~T}$ with length equal to $5000 \mathrm{~mm}$ and diameter equal to $45 \times 3.5 \mathrm{~mm}$. Acoustic emission sensor GT301 is fixed on one of the pipe's ends.

Nozzle with calibrated aperture arranged on the pipe as part of the special tool acts as the transmitter of stress waves (leak imitator). Noise emitted during gas discharge from the nozzle imitates the noise of water leakage. Gas supply system consists of pressurized gas cylinder, gas pressure regulator, measuring volume, pressure gauge measuring gas pressure inside the measuring volume and the system of valves and nozzle.
Procedure for calibration of gas flow channel consists of the measurement of the value of gas pressure imitating the working medium.

Measuring volume $V$ from which gas is bled through the nozzle is filled from the pressurized gas cylinder. Ultrasonic oscillations exciting Rayleigh surface waves are generated in the process of gas bleeding. Average gas flow rate is calculated according to the following formula:

$$
G=-V M_{\mathrm{N}}\left(V_{\mu} P_{0}\right)^{-1} \times d P / d t
$$

where $G$ is the gas flow rate, $\mathrm{kg} / \mathrm{s} ; M_{\mathrm{N}}$ is the molecular mass of the gas, $\mathrm{kg} ; V_{\mu}$ is equal to $22,4 \times 10^{-3} \mathrm{~m}^{3} ; P_{0}$ is the value of atmospheric pressure, $\mathrm{MPa} ; d P / d t$ is the rate of pressure reduction, $\mathrm{MPa} / \mathrm{s}$.

Let us note that the above formula is valid only for the subcritical gas discharge i.e. for the value $P \leq 2$ atmospheres. Above this value pressure changes linearly while the flow rate remains constant. Nevertheless, formula (1) determines gas flow rate specifically in the area of small values which allows testing sensitivity of the automated measurement system with regard to small leaks.

Dependences of the value of pressure in the measuring volume and gas flow rate on the time of gas discharge are presented in Figure 1. Gas discharge in the present experiment begins from 12-th second. Value of gas flow rate has physical meaning from approximately 100-th second.

\section{Traditional approach}

Two integral statistical characteristics - acoustic wave spectrum power and average frequency of the spectrum - are usually applied for the detection of leaks during diagnostics of pipelines by acoustic method.

Spectrum power is equal to:

$$
M=\sum_{i=1}^{N} S\left(F_{i}\right),
$$

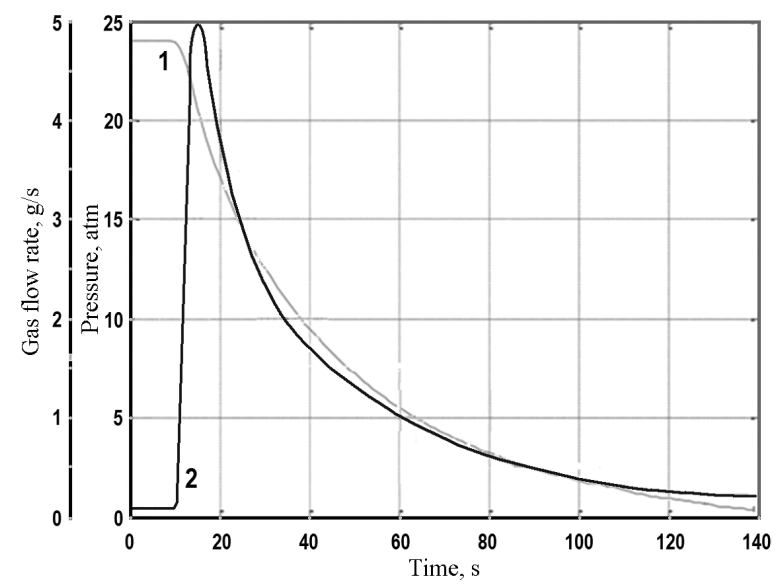

Figure 1. Time dependences of pressure and gas flow rate during gas discharge: 1 - pressure; 2 - flow rate. 
where $S\left(F_{i}\right)$ is the spectral power density (SPD) on a frequency $F_{i}$ proportional to the area under the SPD curve and determined by the dispersion of the measured acoustic signal.

Average spectral frequency $F_{R}$ (Reiss frequency)

$$
F_{R}=\sum_{i=1}^{N} F_{i} \cdot S\left(F_{i}\right) / \sum_{i=1}^{N} S\left(F_{i}\right)
$$

is determined by the shape of frequency spectrum of the measured acoustic signal.

Application of the above diagnostic indicators is predetermined by the following facts:

- Acoustic spectrum power increases with growing leakage;

- Shape of acoustic spectrum changes when the leak emerges as compared with the background value (sharp shifting of the center of mass of the acoustic spectrum in the area of low frequencies takes place during the initial phase of leak initiation);

- Implementation of estimation of the value and tentative location of the leakage source is possible using the correlation between quantitative characteristics, i.e. the diagnostic indicators, characterizing variation of power and shapes of spectra for sensors installed in different points.

Dispersions and average frequencies of acoustic signal of the sensor are presented in Figure 2. It is evident that beginning from 500-th second with gas flow rate equal to about $0.04 \mathrm{~g} / \mathrm{s}$ these diagnostic indicators practically fail to identify the leak.

Ratio of signal energies on different parts of the power spectrum, as well as the ratio of current average frequency to the average background frequency were selected as the diagnostic indicators in the suggested approach. Since signal within different frequency bands is attenuated differently, assumption was made that ratios of energies of signals within different frequency bands will be more informative diagnostic indicators than the total dispersion.

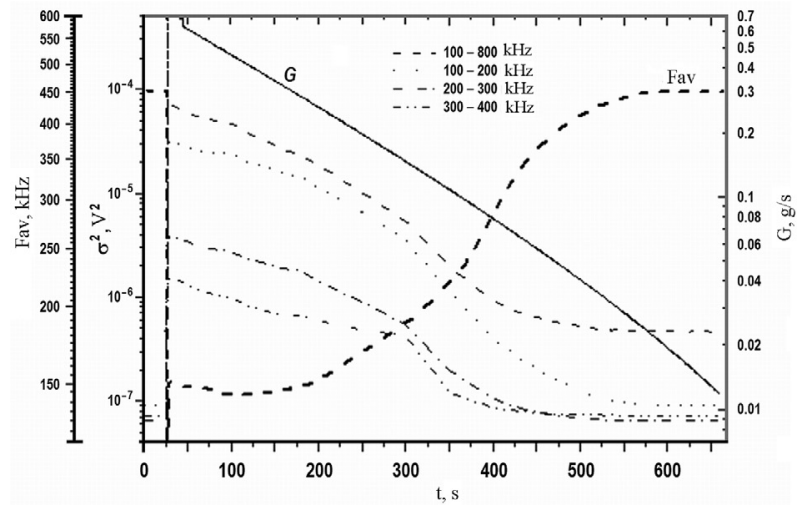

Figure 2. Average frequencies and dispersions of acoustic sensor signal within the frequency range from $100 \mathrm{kHz}$ to $800 \mathrm{kHz}$.
Signal energies within the frequency band from 100 $\mathrm{kHz}$ to $200 \mathrm{kHz}\left(E_{1}\right)$, from $200 \mathrm{kHz}$ to $300 \mathrm{kHz}\left(E_{2}\right)$, from $300 \mathrm{kHz}$ to $400 \mathrm{kHz}\left(E_{3}\right)$ and over the whole spectrum from 0 to $800 \mathrm{kHz}\left(E_{4}\right)$, as well as the average frequency of the spectrum $F_{R}$ were calculated. The above values were calculated by averaging the signal over the time interval equal to one second. Measurement discretization frequency $F_{s}=2 \mathrm{MHz}$. Diagnostic indicators were constructed based on these values as follows: different ratios $P_{i}=E_{i_{1}} / E_{i_{2}}$ for $i=1-8\left(i_{1}\right.$ and $i_{2}$ are the different combinations of partial energies) were taken, as well as $P_{9}=$ $F_{R} / F_{\text {bgr }}$.

It was necessary to normalize all indicators according to the same scale for the purpose of performing their analysis and to construct covariance matrix. In order to achieve the above the following formula was applied to each of the indicators:

$$
P^{*}=(P-\mu) / \sigma
$$

where dispersion $\sigma$ and mathematical expectation $\mu$ are calculated for the current time window.

Scaled indicators are shown in Figure 3. Their difference from the background values is significantly better pronounced in such representation. They practically coincide with background only by the 640 -th second of the leakage.

Let us construct covariance matrix for the indicators at $i$-th time moment

$$
C_{i j}=\sum_{j=1}^{N} P_{i j}^{*} \times P_{i j}^{* T}-M_{i} \times M_{i}^{T},
$$

And, following this, calculate its eigenvalues which are, essentially, the dispersions of the indicators in the new space:

$$
\Lambda Q=\mathbf{C} Q
$$

where $\Lambda=\operatorname{diag}\left(\lambda_{1}, \lambda_{2}, \ldots, \lambda_{N}\right)$ are the eigenvalues of the covariance matrix; $Q=\left(q_{1}, \ldots, q_{N}\right)$ are the respective eigenvectors of the covariance matrix.

For calculating covariance matrix all indicators $P_{i}$ were averaged within the frame of the current time window

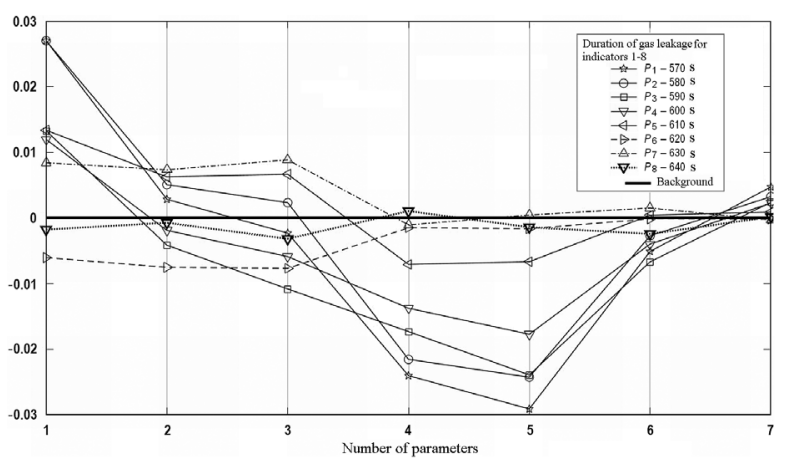

Figure 3. Normalized diagnostic indicators of the acoustic sensor at different time moments. 
which was equal to 10 seconds, i.e. indicators $P_{i j}$ were taken at each time moment for the last $10 \mathrm{~s}$, where $i$ is the number of the indicator, and $j$ is its time stamp within the limits of the current time window. It is specifically averaging over the time window which is meant by the summation in Formula (3).

Comparison of variation of the largest eigenvalue of the covariance matrix (Fukunaga 1972) (LEVCM) with time with signal dispersions, as well as leakage flow rate in $\mathrm{g} / \mathrm{s}$ is shown in Figure 4. It is evident from the figure that application of LEVCM as the indicator for leak control is found to be more sensitive than the signal dispersion or the average spectrum frequency. Indication of the leak when LEVCM is applied occurs at flow rate $\sim 0.015$ $\mathrm{g} / \mathrm{s}$, while when indication of leak is made using signal dispersion it is achieved at flow rate $\sim 0.04 \mathrm{~g} / \mathrm{s}$.

Thus, indication of leak using LEVCM allows enhancing sensitivity of leak detection by more than two times. Results of leak indication are presented in Figure

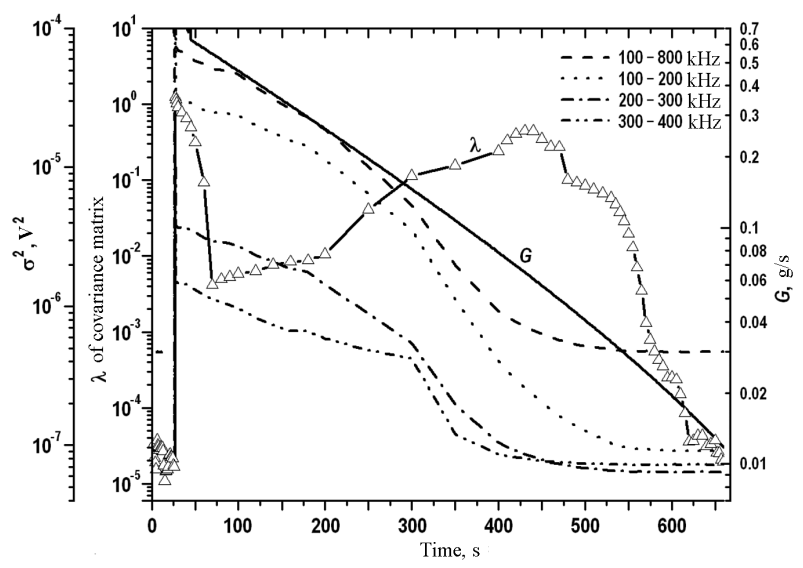

Figure 4. Comparison of variation of the largest eigenvalue of covariance matrix (LEVCM) in time with signal dispersions (Fukunaga 1972).

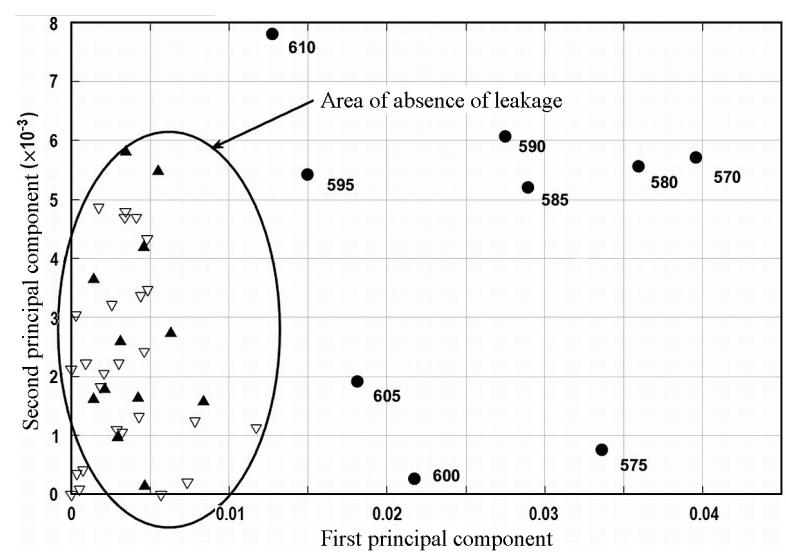

Figure 5. Tome evolution of the leak in the representation of principal components of the covariance matrix: triangles - conditions with absence of leak (vertex down - before initiation of the leak, vertex up - after leak attenuation); solid circles - conditions of leak at respective time moments.
5 in the representation of principal components of the covariance matrix.

The fact is of interest that LEVCM has sufficiently high value in the area of coolant flow rate where traditional diagnostic indicators (dispersion and average spectral frequency) already begin to attenuate exponentially. Therefore, it can be expected that in the presence of external noises (interferences) the method implying the use of LEVCM will demonstrate much higher sensitivity of leak detection than the conventional diagnostic indicators.

The following test problem was solved for confirming this fact. White noise signal with dispersion equal to $1 \times 10^{-2} \mathrm{~V}^{2}$ was added to the signal imitating the leak.

Comparison of variation of the largest eigenvalue of covariance matrix (LEVCM) with time with dispersions of signals and average spectral frequency is shown in Figs. 6 and 7. It is clear from the figure that application of LE$\mathrm{VCM}$ as the indicator significantly improves sensitivity of leak detection as compared with the use of dispersion of signal or average spectral frequency. Leak detection occurs in the case of application of LEVCM at the low value of rate equal to $\sim 0.02 \mathrm{~g} / \mathrm{s}$, while in the case of leak detection using signal dispersion of average spectral frequency

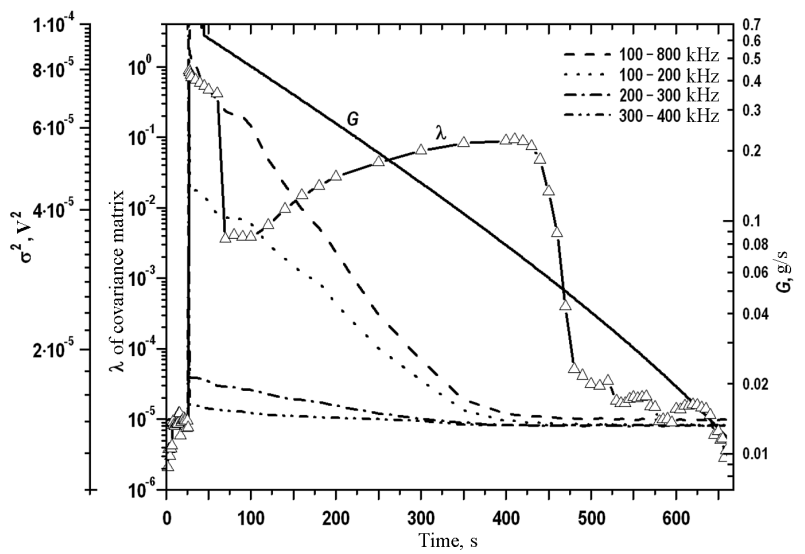

Figure 6. Comparison of time variation of LEVCM with signal dispersions for the case when white noise signal is superimposed.

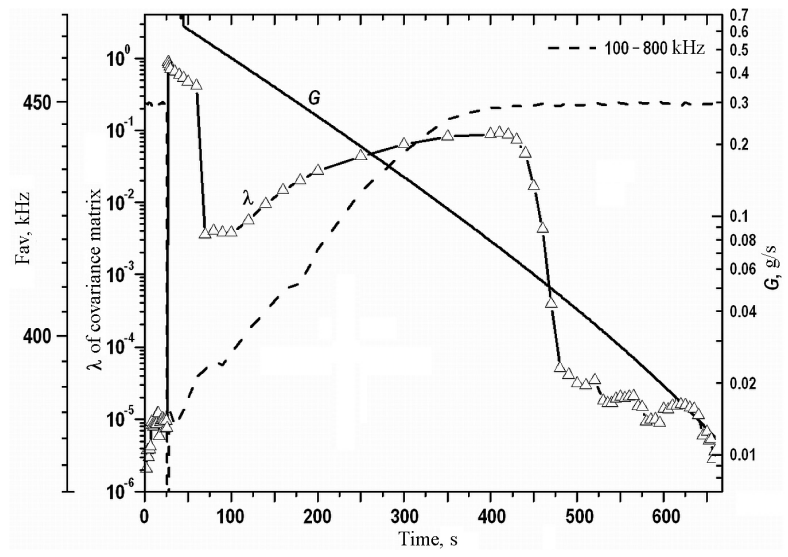

Figure 7. Comparison of time variation of LEVCM with average signal frequencies. 
occurs at $\sim 0.1 \mathrm{~g} / \mathrm{s}$. That means that when LEVCM is used sensitivity increases by approximately five times.

Practical application of the suggested approach in operable leak control systems can be implemented with employment of broadband acoustic sensors.

\section{Conclusion}

The suggested new approach allows enhancing sensitivity of leak control systems with regard to low-volume leaks

\section{References}

- Abagyan AA, Dmitriev VM, Klebanov LA, Kroshilin AE, Larin EP, Morozov SK (1987) System for monitoring and diagnostics of NPP unit operational conditions. Atomnaya energiya 63: 311-315. [in Russian]

- Arkadov GV, Pavelko VI, Finkel BM (2010) Systems diagnosis of VVER. Energoatomizdat Publ., Moscow, 391 pp. [in Russian]

- Basseville M (1988) Detecting changes in signal and systems - a survey. Automatica 24(3): 309-326. https://doi.org/10.1016/00051098(88)90073-8

- Frank PM (1990) Fault diagnosis in dynamic systems using analytical and knowledge-based redundancy - a survey and some new results. Automatica, 26(3): 459-474. https://doi.org/10.1016/00051098(90)90018-D

- Fukunaga K (1972) Introduction to Statistical Pattern Recognition. Academic press, New York and London, 375 pp.

- Herbert MR (1984) A review of on-line diagnostic aids for nuclear power plant operators. Nucl. Energy 23(4): 259-264.

- Iserman R (1984) Process fault detection based on modeling end estimation methods - a survey. Automatica 20(4): 387-404. https://doi org/10.1016/0005-1098(84)90098-0

- Leskin ST (1997) Algorithm development for detecting abnormality of NPP equipment conditions based on technological testing results. Izvestiya vuzov. Yadernaya energetika 1997(4): 4-12. [in Russian]

- Leskin ST, Slobodchuk VI, Shelegov AS (2016) Analysis of VVER1000 main circulation pump conditions in operation. Isvestya vuzov. Yadernaya energetika 2016(4): 12-22. [in Russian]

- Leskin ST, Slobodchuk VI, Shelegov AS, Kashin DYu (2017) Analysis of safety system pumps conditions based on their testing although it requires experimental investigation of the case of real leaks of heated coolant.

In the case of presence of background noises sensitivity of the suggested method with respect to small leaks increases by up to five times as compared with conventional approach.

Implementation of the suggested approach will not require significant expenditures for upgrading existing leak control systems operated at present on different NPPs.

results. Isvestya vuzov. Yadernaya energetika 2017(1): 42-50. [in Russian]

- Patton R, Frank P, Clark R (1989) Fault Diagnosis in Dynamic Systems - Theory and Applications. Prentice Hall Inc., Englewood Cliffs, 360 pp.

- Pavelko VI (1990) Review of applications of expert methodology systems for nuclear power engineering. Atomnaya tekhnika za rubezhom 1990(11): 1-8. [in Russian]

- Reisen C, Marshall E (1988) Evaluating operator support system in realistic conditions at hammlab. Nuclear Engineering International, 33(402): 39-41.

- van Ryzin V (1980) Classification and Clustering. MIR Publ., Moscow, 365 pp. [in Russian]

- Tao Gu, Tou JT (1982) A new criterion for optimal classification. Pattern Recognition 2: 1063-1065.

- Tu J, Gonsales R (1978) Pattern Recognition Principles. MIR Publ., Moscow, 412 pp. [in Russian]

- Ujita Hiroshi (1986) A probabilistic analysis method of evaluate the effect of human factors on plant safety. Nucl. Tehnol. 76(3): 370-376.

- Urig Robert E (1991) Potential application of nuclear networks to nuclear power plants. Proc. Amer. Power Conf. V. 53. Pt. 2 53-rd. Annual Meet., Chicago, III., Apr. 29-May 1, 946-951.

- Weiss S, Reagan W, Roe J (1988) Experience with operator aids for nuclear power plants in the USA. Proc. Intern. Conf. on Man-Machine Interface in Nuclear Industry. Tokyo, 15-19 Feb., Vienna, 323-329.

- Willsky AS (1976) A Survey of design methods for failure detection in dynamic systems. Automatica 12: 601-611. https://doi. org/10.1016/0005-1098(76)90041-8 